\title{
Adverse Drug Reactions to Antiretroviral Therapy (ART): Prospective Study in HIV Infected Adults in Sikasso (Mali)
}

Aboubacar Alassane Oumar*, Amadou Abdoulaye, Mamoudou Maiga, Younoussa Sidibé, Yacouba Cissoko, Issa Konaté, Maimouna Diarra, Fanta Sangho, Jean Paul Dembélé, Paul M Tulkens and Sounkalo Dao

University of Sciences, Techniques and Technology Bamako, Bamako, Mali

"Corresponding author: Aboubacar Alassane Oumar, University of Sciences, Techniques and Technology Bamako, Bamako, Mali, Tel: 00223 20226786; E-mail: alabmai@yahoo.fr

Received date: Apr 06, 2017; Accepted date: Apr 19, 2017; Published date: Apr 27, 2017

Copyright: (C) 2017 Oumar AA, et al. This is an open-access article distributed under the terms of the Creative Commons Attribution License, which permits unrestricted use, distribution, and reproduction in any medium, provided the original author and source are credited.

\section{Abstract}

Objective: The purpose of this study was to evaluate the adverse effects of Antiretroviral Therapy (ART) in adult HIV-infected patients in decentralized setting of Sikasso, in Mali.

Methods: This is a proactive study that took place from 2 January 2011 to 30 December 2012 at the Hospital of Sikasso (Department of Medicine). HIV-infected adult patients who have started ART at least 3 months before were included in this study to monitor the laboratory and clinical Adverse Drug Reactions (ADR) for at least 6 months. The WHO classification of adverse drug reactions has been used to investigate the causality of antiretrovirals.

Results: Women were the most represented with $58 \%$ of the cases. The most represented age range was $26-47$ years with $73.6 \%$. Of the 178 patients enrolled, $61.2 \%$ had an ADR. ADR were neurological (40.4\%), digestive $(35.8 \%)$, cutaneous (18.3\%) and hematological (5.5\%). Stavudine was the most incriminated molecule, in $24.8 \%$ of the cases. The WHO grade 4 classification of ART toxicity was represented in $3.4 \%$ of the cases. The WHO causality score of "certain" was found in $29.8 \%$ of the cases.

Conclusion: Adverse effects of Antiretrovirals are frequent and could be life-threatening in short and long terms. Regular follow-up of patients receiving these triple therapies, and the associated complications, is essential. We recommend active surveillance of antiretroviral therapy to strengthen Pharmacovigilance in Mali.

Keywords: Adverse effects; Sikasso; Mali; Pharmacovigilance

\section{Introduction}

Acquired immunodeficiency syndrome or AIDS is the serious consequence of infection due to the Human Immunodeficiency Virus (HIV) and is a major public health problem [1]. In Mali, the prevalence of HIV/AIDS is $1.1 \%$ among people aged 15 to 49 with an estimated 66 392 people in 2012 [2]. Antiretrovirals (AR) provide low viral load and maintain the immune system by raising the number of TCD4+lymphocytes. They reduce the incidence of complications, improve health and prolong patient survival [3]. Knowledge about the toxicity associated with antiretrovirals has improved significantly with the accumulation of experience of long-term patient therapy combinations [4]. Despite these successes, patients are still exposed to the toxicities of these antiretroviral molecules. The adverse effects of AR drugs are indeed a common reason for discontinuation of treatment in infected patients. More than $25 \%$ of patients discontinue treatment in the first year because of these effects [5]. These adverse effects result in changes in biological parameters with often clinical manifestations [6], which can affect many organs of the body. The most common adverse reactions are dermatological reactions and Gastrointestinal (GI) disorders [7]. Hepatic toxicity occurs in about one-third (1/3) of HIV-infected patients after initiation of therapy [8]. Metabolic complications are frequently reported, such as lipodystrophy, hyperinsulinemia/hyperglycemia and osteoporosis, osteonecrosis associated with Protease Inhibitors (PIs), and lactic acidosis with Nucleoside Reverse Transcriptase Inhibitors (NRTI) $[7,9]$. Dyslipidemia associated with an increased risk of cardiovascular disease is also reported in HIV-1 infected patients who receive ARV [10]. Several other rare but serious adverse reactions are also reported: Anemia associated with zidovudine or Azidothymidine (AZT); Stavudin-induced peripheral neuropathy $(\mathrm{d} 4 \mathrm{~T})$, toxicity of retinoids associated with protease inhibitors (pruritus and ingrown toenails) and hypersensitivity reactions associated with Non-Nucleoside Reverse Transcriptase Inhibitors (NNRTIs)]. All of these adverse effects can have a significant impact on patients' quality of life and adherence to treatment. Adverse events in HIV-infected patients are well documented in developed countries such as Belgium [11,12]. In Mali, very few studies on adverse effects and their management have been performed $[13,14]$. What motivated us in choosing this topic: The adverse effects of AR in HIV-infected adult patients and the evaluation of the management of these Adverse Drug Reactions (ADR)? Our goal was to describe the frequency, nature and nature of adverse events in a decentralized cohort of adult patients in Sikasso, Mali.

\section{Materials and Methods}

Our study was conducted at the Kénédougou Solidarity Research Center (CERKES). The study was conducted from January 2, 2011 to December 30, 2012. This was a prospective cohort study based on the records of patients infected with HIV and AR therapy. Each naive patient who was to receive antiretroviral treatment in the CERKES medical department had an individual medical record. This file 
included a part for the initiation of treatment and follow-up forms. After the first prescription of antiretrovirals, the patients were reviewed at D15, M1, M3 and M6. At each control visit, careful physical examination and examination allowed clinical events to be identified, including those related to the use of drugs. Systematic patient prescriptions included blood count, CD4 cell counts, blood glucose, creatinine, cholesterol, triglycerides, and transaminases. The study was performed only on patients meeting our inclusion criteria. These inclusion criteria were: patients aged 15 years and over; patients under AR treatment with CERKES; presence of adverse reactions after antiretroviral treatment or No. The Adverse Drug Reaction (ADR) refers to adverse or unwanted responses or responses in humans when taking a recommended dose for prophylactic, diagnostic or therapeutic purposes. In our work, the ADR was the set of symptoms reported by the patient or reported by the physician and related to the use of AR. A sign was considered ADR if it appeared in the patient after the initiation of treatment, that there was not another obvious etiology to explain its occurrence and whether it was reported in the literature as Adverse Drug reactions possible of one of the molecules used. Intensive adverse drug reactions research was observed during the study. The WHO classification of adverse reactions has been used to investigate the causality of antiretrovirals [15]. The use of antiretrovirals as a treatment for Human Immunodeficiency Virus (HIV) began in 1987 with the commercialization of zidovudine (AZT). Several other molecules of the same therapeutic class (nucleoside reverse transcriptase inhibitors or NRTI) were then developed. Since 1996, two other classes of antiretroviral drugs, namely Protease Inhibitors (PIs) and Non-Nucleoside Reverse Transcriptase Inhibitors (NNRTI), have been introduced for the introduction of "highly active" therapies (HAART: Highly Active Antiretroviral Therapy) improving the management of infected patients and significantly reducing patient morbidity and mortality. Antiretroviral therapy consisted of the following regimens:

- Stavudine+lamivudine+nevirapine

- Zidovudine+lamivudine+nevirapine

- Tenofovir+emtricitabine+efavirenz

- Zidovudine+lamivudine+Efavirenz

- Stavudine+lamivudine+efavirenz

- Zidovudine+lamivudine+lopinavir/ritonavir

Thanks to the many possible combinations, these molecules allow better control of the disease by reducing viral replication at an undetectable rate in the vast majority of cases (less than 50 copies $/ \mathrm{mL}$ ). However, these treatments still have many adverse effects and are incapable of eradicating the virus, requiring lifetime administration. The data were collected on survey forms and then entered and analyzed on the SPSS 16.0 software. Confidentiality was paramount; informed consent was always requested prior to inclusion in the study. The results obtained have been communicated to the administrative authorities of CERKES. This study was approved by the ethics committee of the Faculty of Medicine of Bamako.

\section{Results}

Our study involved 178 HIV-infected patients at CERKES in Sikasso. The most represented age group was $26-36$, i.e., $41.6 \%$ of cases with an average age of 35.2 years and extremes between 16 and 62 years. The majority of our patients were female (57.8\%) (Figure 1).
ADR versus $81.1 \%$ for patients without ADR. The majority of our patients had a CD4 cell count of less than 350 cells $/ \mathrm{mm}^{3}$ with $89.9 \%$ in patients with ADR versus $84 \%$ in patients without ADR. Hepatitis, high blood pressure (hypertension) and diabetes were the associated conditions in patients with $\mathrm{ADR}$ against tuberculosis in patients without ADR.

\section{Distribution of Sex}

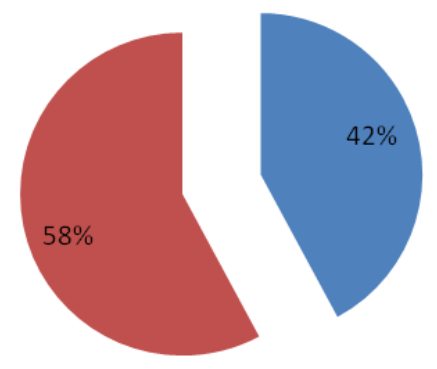

nale

- Female

Figure 1: Distribution of patients by sex.

The most commonly used therapeutic combination consisted of two NRTIs plus one NNRTI 97.3\% in patients with ADR versus $100 \%$ in patients without ADR. The most common treatment regimen was the combination of $\mathrm{D} 4 \mathrm{~T}+3 \mathrm{TC}+\mathrm{NVP} 60.5 \%$ in patients with ADR versus $65.2 \%$ in patients without ADR. The majority of our patients were observed $88.1 \%$ in patients with ADR versus $94.2 \%$ in patients without ADR. Some patients had a change in the regimen for ADR (14.7\%) (Table 1$)$. The majority of reported ADR were WHO grade $1(43.8 \%)$ (Figure 2).

The mean time to onset of ADR after treatment was $26.9 \pm 42.4$ days ( 1 to 180 days) (Table 1 ). In the first 7 days of treatment, ADR occurred in $10 \%$ of cases; $39 \%$ between $8: 15$ days; $51 \%$ after 16 days. Approximately $40 \%$ of ADR were reported before the first 100 days of treatment (Figure 3).

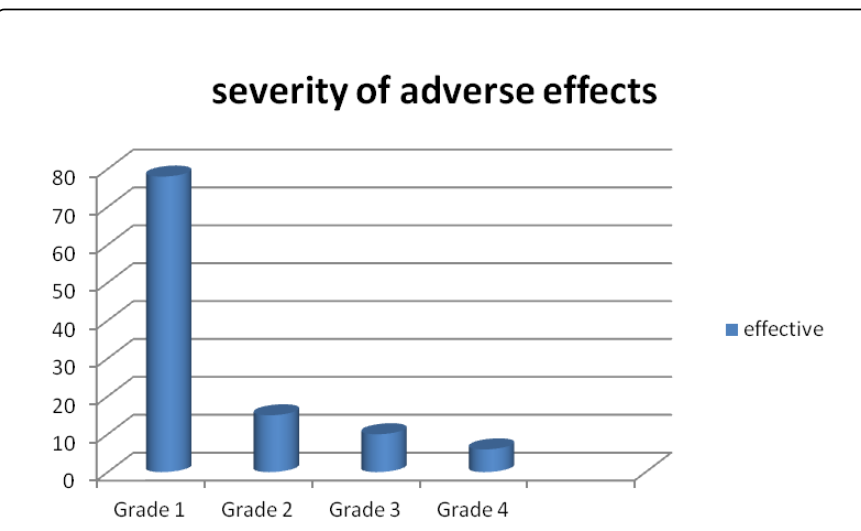

Figure 2: Distribution of patients by grade of adverse reactions according to WHO.

The causality of adverse effects according to the WHO score was used to determine a "certain" causality score in $29.8 \%$ of the cases 
Citation: Oumar AA, Abdoulaye A, Maiga M, Sidibé Y, Cissoko Y, et al. (2017) Adverse Drug Reactions to Antiretroviral Therapy (ART): Prospective Study in HIV Infected Adults in Sikasso (Mali). J Pharmacovigil 5: 228. doi:10.4172/2329-6887.1000228

(Table 2). The ADR were neurological disorders (40.4\%) and digestives (35.8\%) (Table 3).

The $\mathrm{D} 4 \mathrm{~T}+3 \mathrm{TC}+\mathrm{NVP}$ regimen was the most associated with ADR with $36.7 \%$ of the cases (Table 2). The management of the notified ADR consisted of: advices ( 85 patients, $78 \%$ of cases), symptomatic treatment (28 patients, $25.7 \%$ ), change of a molecule of the treatment regimen (16 patients, 14.7\%). Adverse Drug reactions were favorable in 108 patients (99.1\% of cases). Only one case required follow-up. The majority of neurological disorders were unlikely (56\%) (Table 4$)$.

\begin{tabular}{|c|c|c|c|}
\hline \multicolumn{2}{|l|}{ Factors } & \multirow{2}{*}{$\begin{array}{l}\text { Patients with ADR } \\
36.3\end{array}$} & \multirow{2}{*}{$\begin{array}{l}\text { Patients without ADR } \\
33.5\end{array}$} \\
\hline Age & Mean & & \\
\hline Level CD4 & Mean & 189.7 & 168.9 \\
\hline \multirow[t]{3}{*}{$\mathrm{CD} 4$} & Tranche de CD4 & - & - \\
\hline & $<350$ & 98 & 58 \\
\hline & $\geq 350$ & 11 & 11 \\
\hline \multirow[t]{2}{*}{ Residence } & Sikasso & 68 & 53 \\
\hline & Off Sikasso & 41 & 16 \\
\hline \multirow[t]{4}{*}{ WHO clinical stage } & Stade 1 & 1 & 0 \\
\hline & Stade 2 & 52 & 13 \\
\hline & Stade 3 & 48 & 56 \\
\hline & Stade 4 & 8 & 0 \\
\hline Associated pathologie & Hepatitis & 2 & 0 \\
\hline Diabetes & 1 & 0 & \\
\hline Tuberculosis & 0 & 13 & \\
\hline \multirow[t]{2}{*}{ Protocole } & $2|N T I+1| N N T I$ & 106 & 69 \\
\hline & $2 I N T I+1 \mid P$ & 3 & 0 \\
\hline \multirow[t]{6}{*}{ Scheme } & $\mathrm{D} 4 \mathrm{~T}+3 \mathrm{TC}+\mathrm{NVP}$ & 66 & 45 \\
\hline & $A Z T+3 T C+N V P$ & 31 & 4 \\
\hline & $\mathrm{TDF}+\mathrm{FTC}+\mathrm{EFV}$ & 0 & 13 \\
\hline & $A Z T+3 T C+E F V$ & 7 & 4 \\
\hline & $\mathrm{D} 4 \mathrm{~T}+3 \mathrm{TC}+\mathrm{EFV}$ & 2 & 3 \\
\hline & $A Z T+3 T C+L P V / r$ & 3 & 0 \\
\hline \multirow[t]{2}{*}{ Modified Scheme } & YES & 16 & 0 \\
\hline & No & 93 & 69 \\
\hline \multirow[t]{2}{*}{ Observance } & YES & 96 & 65 \\
\hline & No & 13 & 4 \\
\hline Time of occurrence & Mean (day) & 26.97 & \\
\hline
\end{tabular}

Table 1: Patient characteristics. 
Citation: Oumar AA, Abdoulaye A, Maiga M, Sidibé Y, Cissoko Y, et al. (2017) Adverse Drug Reactions to Antiretroviral Therapy (ART): Prospective Study in HIV Infected Adults in Sikasso (Mali). J Pharmacovigil 5: 228. doi:10.4172/2329-6887.1000228

Page 4 of 7

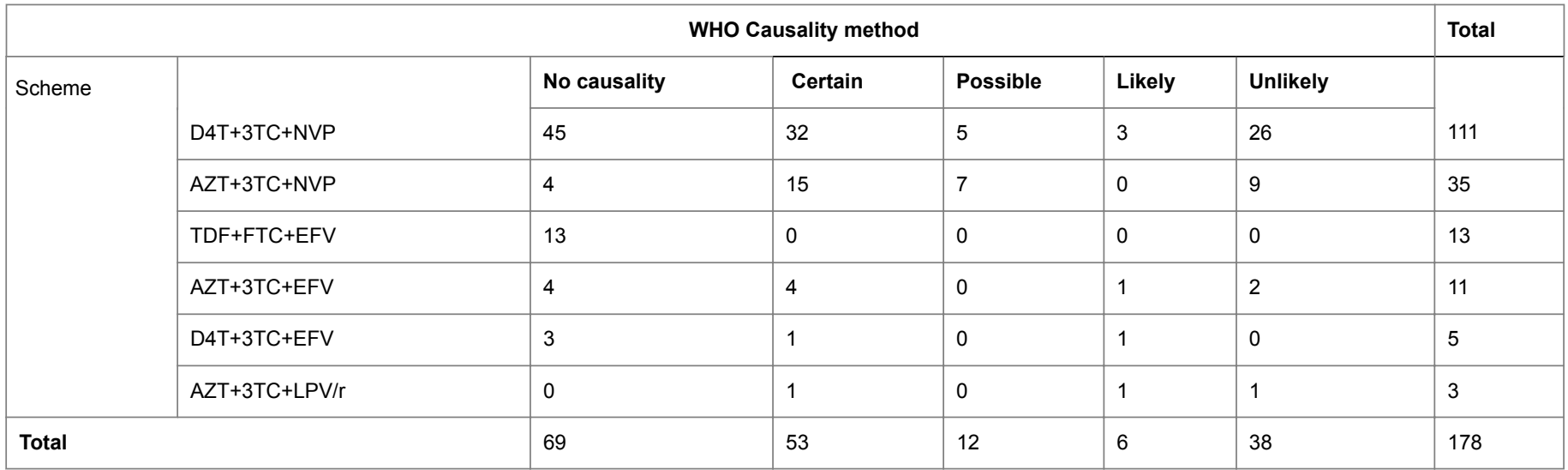

Table 2: Relationship between schemas and the WHO causality method.

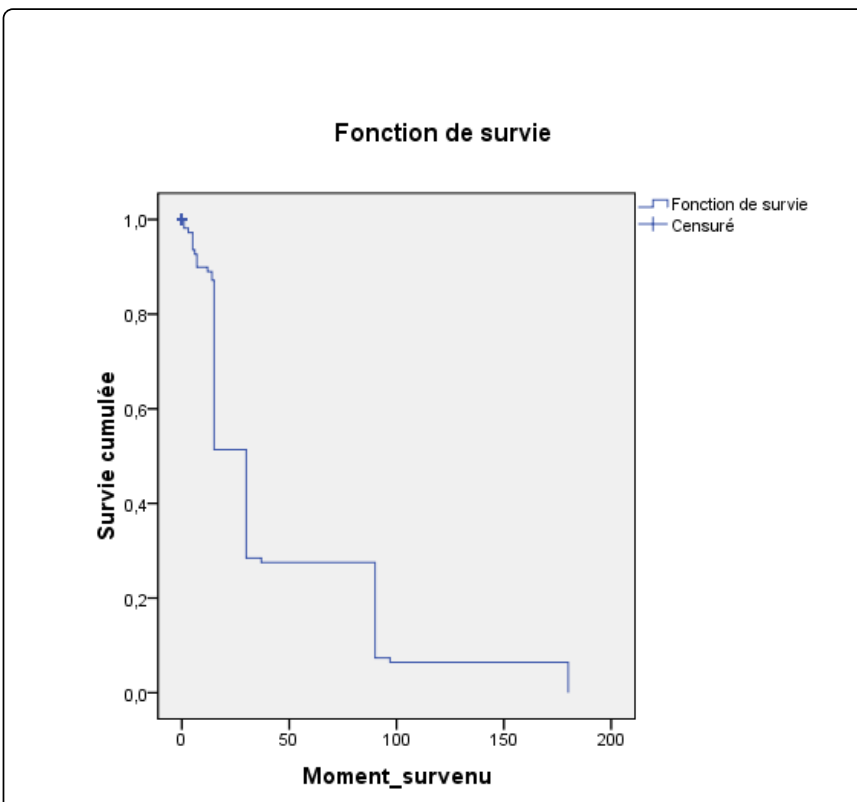

Figure 3: Kaplan Meier curve of time of occurrence of adverse reaction.

\begin{tabular}{|l|l|l|}
\hline Types of adverse effects & Effective & Percentage \\
\hline Digestive disorders & 39 & 35.8 \\
\hline Skin disorders & 20 & 18.3 \\
\hline Neurological disorders & 44 & 40.4 \\
\hline Hematologic disorders & 6 & 5.5 \\
\hline Total & 109 & 100 \\
\hline
\end{tabular}

Table 3: Distribution according to the type of adverse effects.

\section{Discussion}

Our descriptive and analytical study with a prospective data collection was carried out to evaluate adverse effects in people living with HIV/AIDS under antiretroviral treatment at CERKES in Sikasso.
Many difficulties were encountered: Changes in therapeutic protocol due to the withdrawal of the combination $(\mathrm{d} 4 \mathrm{~T}+3 \mathrm{TC}+\mathrm{NVP})$ on the Malian market, the irregularity of patients on visits, the temporary or permanent unavailability of certain complementary examinations: CD4, viral load in Sikasso. Despite these difficulties we were able to obtain the complete results of 178 patients under antiretroviral and regular treatment in follow-up. The sample consisted predominantly of women with an average age of 36.3 years versus 33.5 years. In Ghana, Lartey et al. found a female predominance in their study [16]. This female predominance can be explained by the frequency of heterosexual transmission and the biological vulnerability of women with a larger contact area and a longer time. Similarly, women's socioeconomic vulnerability places women at greater risk of transmission. The difference between the countries of the North and South finds two explanations: On the one hand the predominant heterosexual transmission in the South associated with female vulnerability. On the other hand, the relatively high role of homosexuality and the use of injectable drugs in the North in the transmission of the disease. In Senegal, Etard et al. [17], found a sample of 404 patients on ARV with an average age of 37 years \pm 6 . Studies on the ARs of ARVs performed in Mali [14], Cotonou [18] and Iran [19] noted an average age of 35 years, 41 years and 36 years, respectively, with a female predominance. On the other hand, studies carried out in developed countries indicate a male predominance $[20,21]$. The pathologies associated with our patients were hepatitis, hypertension and diabetes. Guitton et al. found hepatitis influences on ADR in HIV patients [22]. Tuberculosis was most prevalent in patients without ADR. Khalili et al, found cases of co-infections with tuberculosis in 8\% [19]; Modayil et al. in 2010 found $39.7 \%$ of cases of co-infections with tuberculosis [23]. Tuberculosis is a pathology that could be associated and be an additional risk. The mean CD4 count was 189.7 cells $/ \mathrm{mm}^{3}$ vs. 168.9 cells $/ \mathrm{mm}^{3}$. Our results are comparable to those obtained by Maiga et al. [24] in Mali, and those of Bennett et al. [25] in the USA. These data show a severe immunosuppression resulting from a very late diagnosis. Furthermore, there was no association between the level of CD4 and the onset of ADR $(p=1.26)$. The most commonly used therapeutic combination consisted of two NRTIs plus one NNRTI. In addition, NNRTI based regimens have been widely recommended by WHO for African countries, in addition have demonstrated efficacy at least equal to that of IP based associations and the advantage of NRTIs is the ease of compliance due to the small number of tablets to be swallowed, a smaller proportion of long-term complications, mostly metabolic [26]. Although effective, these molecules have a low genetic barrier, hence a higher precociousness of the emergence of resistant mutants. The latest 
Page 5 of 7

recommendations in France advocate the use of a ritonavir boosted anti-protease in first-line treatment of HIV-infected patients due to a higher genetic barrier of protease inhibitors compared to NNRTI [27]. Indeed, in our sample, the combination of $\mathrm{d} 4 \mathrm{~T} / 3 \mathrm{TC} / \mathrm{NVP}$ predominated in the prescription of triple therapy at inclusion. The frequent use of $\mathrm{d} 4 \mathrm{~T} / 3 \mathrm{TC} / \mathrm{NVP}$ was in line with the Malian recommendation for the care of people living with HIV in 2012 [28]. Of the 178 patients, $61.2 \%$ had at least one adverse drug reactions. Khalili et al, respectively found $87.6 \%$ in their study [19]. Clinical adverse effects of ARVs, mainly digestive disorders, have been reported during antiretroviral therapy by several authors $[18,19]$. The same was true regardless of age, history, clinical stage, and CD4 count at baseline. ADR had occurred in the average 26.9 days. Treatment of clinically evaluated ADR consisted of counseling (78\%). Modayil et al., Khalili et al., found similar results $[19,23]$. Our study summarized the dominant complaints of patients and observations of physicians following antiretroviral therapy. It was not possible to account for the long-term complications of ARV such as lipodystrophy. It is currently described in Western countries as the most common adverse reaction. This is currently described in western countries as the most annoying side effect [29].

\begin{tabular}{|l|l|l|l|l|l|}
\hline Effets indésirables & N & Certaine & Possible & Likely & Unlikely \\
\hline Digestive disorders & 39 & 20 & 7 & 4 & 8 \\
\hline Skin disorders & 20 & 12 & 2 & 0 & 6 \\
\hline Neurological disorders & 44 & 15 & 2 & 2 & 25 \\
\hline Hematologic disorders & 6 & 6 & 0 & 0 & 0 \\
\hline
\end{tabular}

Table 4: Relationship between causality and adverse effects.

Some cases have been described in the African cohort studies [30]. Our study provides guidance to guide young prescribers in an African context. Other authors have found similar results $[18,19]$. The frequency of ADR was significantly different according to the patterns used. In the majority of cases, ADR were observed at the beginning of treatment and were transient. The time of occurrence varied considerably depending on the nature of the ADR. Neurosensory ADR were the most frequent (40.4\%) dominated by neuropathy. These results are consistent with those of Zannou et al. in France and Chiffoleau et al. in Cotonou $[18,29]$. The frequency of neurosensory disorders in our study can be explained by the use of stavudine in firstline. Antiretroviral therapy in accordance with national guidelines in Mali. Our results confirm those of several authors on the frequency of neuropathies and risk factors during antiretroviral treatment [31-33]. In other publications, it is the digestive (gastrointestinal) ADR that predominate, observed in $61.1 \%$ of patients in Tunis [34]. The nature of the digestive disorders observed can disrupt patients' appetite and compromise their nutritional status and adherence to treatment. Gastrointestinal disorders therefore deserve special attention during the follow-up of HIV patients. In our study the digestive disorders were dominated by nausea. This result is similar to those obtained by $[14,35,36]$. Pruritus and rash were dermatologic disorders in our patients, these ADR were observed in patients under a protocol including Nevirapine. In a study of Coulibaly et al. on the clinical and biological tolerance of objective Nevirapine $17 \%$ of cases of pruritus [37]. We noted that the regimen containing stavudine was the greatest provider of adverse effects. Stavudine was the origin of peripheral neuropathies that were often irreversible. This ADR was very troublesome for HIV-infected patients. Our results confirm those of several authors on the frequency of neuropathies and risk factors during antiretroviral treatment $[31,33,38,39]$. This observation indicates that the ADR, however frequent and inconvenient, were supported by patients. Oumar et al, in Mali found similar results [13]. The compliance or adherence attitude adopted by these patients may reflect the fact that during the treatment consultation sufficient preparation was made to inform them of the possibility of ADR and the consequences of an interruption of treatment. The therapeutic interruption due to an ADR therefore remains very negligible in our study population $8.9 \%$ of patients. However medication jumps due to ADR are possible, but were not appreciated from the records. Agu and Oparah found $33.9 \%$ of changes due to adverse effects of antiretrovirals [40]. The management of the observed ADR was a function of the severity of the symptoms. In response to minor grade 1 and 2 effects, attention was drawn to the non-serious and transient nature of the symptoms. Baudrant et al. found $88.6 \%$ of minor ADR [20]. The favorable outcome following the treatment offered to patients with ADR is reassuring ( $99.1 \%$ of cases). This explanation was enough to calm the anxiety of the patients. On the other hand, when the effect was severe or persistent despite the symptomatic treatment a change of antiretroviral molecule was made. Similar results have been described in the literature $[19,23,40]$. We used the WHO causality score of adverse drug reactions to assess antiretroviral related causality. The score was "certain" in 29.8\% of the developed Adverse Effects associated with Antiretrovirals. An Iranian study of the adverse effects of antiretrovirals used the same method of the severity grading of adverse reactions of antiretrovirals found a rate varying from 71.4 to $15.6 \%$ according to the types of adverse effects [19]. In Mali, a study carried out in 2011 found a similar result [13].

\section{Conclusion}

The Adverse Drug Reactions of Antiretrovirals are multiple and can be life threatening in the short and long term. An ADR was frequently encountered during our work. The nature of these ADR was predominantly grade 1 and 2 according to the WHO score. The link between Antiretrovirals and ADR was safe according to the WHO scale. The management of ADR consisted of counseling in the majority of cases. Regular follow-up of patients receiving Antiretrovirals triple therapy is essential in order to detect and treat the complications associated with these therapies. We recommend the establishment of a pharmacovigilance system in Mali.

\section{Acknowledgements}

We thank all the patients who participated in this study. This work was presented at the Journées of the French Society of Pharmacology and Therapeutics P2T Angers 2013, Poster N ${ }^{\circ}$ P2_233 and at the 13th Annual Meeting of International Society of Pharmacovigilance ISOP, Pisa 2013 Poster $N^{\circ}$ ISP3447:46. This work benefited from a financier from the University of Bamako, Mali.

\section{Contributions by Authors}

Oumar AA, Dao S, Diarra M, Maiga M, Tulkens P wrote the research protocol and organized the data collection. Diarra $M$, Abdoualye A, Sidibe Y, Konate I, Sangho F, Dembélé JP collected the data. Tulkens $\mathrm{P}$ and Dao S supervised the research study. Oumar AA and Cissoko $\mathrm{Y}$ analyzed and interpreted the results of the study. Oumar AA, Dao S, Maiga M, Cissoko Y, Konate I and Tulknes P 
developed the manuscript. All authors have approved the final version of the manuscript.

\section{References}

1. ONUSIDA (2015) Le point sur l'épidemie du Sida. Geneve: ONUSIDA.

2. Samake S, Traoré SM, Ba S, Dembele E, Diop M, et al. (2012) Demographic Survey of Health in Mali (2012-2013): ICF International p 577.

3. Leclercq P, Roudière L, Viard JP (2004) Complications graves des traitements antirétroviraux. Réanimation 13: 238-248.

4. Lallemand F (1999) Toxicity of antiretroviral drugs. Presse Med 199928 35-36.

5. d'Arminio Monforte A, Lepri AC, Rezza G, Pezzotti P, Antinori A, et al (2000) Insights into the reasons for discontinuation of the first highly active antiretroviral therapy (HAART) regimen in a cohort of antiretroviral naive patients. I.CO.N.A. Study Group. Italian Cohort of Antiretroviral-Naive Patients. Aids 14: 499-507.

6. Fellay J, Boubaker K, Ledergerber B, Bernasconi E, Furrer H, et al. (2001) Prevalence of adverse events associated with potent antiretroviral treatment: Swiss HIV Cohort Study. Lancet 358: 1322-1327.

7. Rudorf DC, SA K (2005) Adverse effects associated with antiretroviral therapy and potential management strategies. J Pharm Pract 18: 258-277.

8. Durrieu G, Lacroix I, Olivier P, Sommet A, Senard JM, et al. (2008) Drugrelated neuropathies: analysis of the French Adverse Drug Reaction Database 1995-2005. Presse Med 37: 935-942.

9. Herman JS, Easterbrook PJ (2001) The metabolic toxicities of antiretroviral therapy. Int J STD AIDS 12: 555-562.

10. Lucas GM, Chaisson RE, Moore RD (1999) Highly active antiretroviral therapy in a large urban clinic: risk factors for virologic failure and adverse drug reactions. Ann Intern Med 131: 81-87.

11. Montessori V, Press N, Harris M, Akagi L, Montaner JS (2004) Adverse effects of antiretroviral therapy for HIV infection. CMAJ 170: 229-238.

12. De Wit S, Sabin CA, Weber R, Worm SW, Reiss P, et al. (2008) Incidence and risk factors for new-onset diabetes in HIV-infected patients: the Data Collection on Adverse Events of Anti-HIV Drugs (D:A:D) study. Diabetes Care 31: 1224-1229.

13. Oumar AA, Dao S, Malle A, Maiga AI, Fongoro S, et al. (2011) Single Center experience with sides effects of "Triomune" in Mali. J Antivir Antiretrovir S3.

14. Oumar AA, Diallo K, Dembele JP, Samake L, Sidibe I, et al. (2012) Adverse drug reactions to antiretroviral therapy: prospective study in children in sikasso (mali). J Pediatr Pharmacol Ther 17: 382-388.

15. Edwards IR, Biriell C (1994) Harmonisation in pharmacovigilance. Drug Saf 10: 93-102.

16. Lartey M, Asante-Quashie A, Essel A, Kenu E, Ganu V, et al. (2014) Adverse drug reactions to antiretroviral therapy during the early art period at a tertiary hospital in Ghana. Pan Afr Med J 18: 25.

17. Etard JF, Ndiaye I, Thierry-Mieg M, Gueye NF, Gueye PM, et al. (2006) Mortality and causes of death in adults receiving highly active antiretroviral therapy in Senegal: a 7-year cohort study. Aids 20: 1181-1189.

18. Zannou DM, Houngbe F, Acapko J, Ade G, Gougounou A, et al. (2007) Undesirable effect of antiretroviral drugs in patients infected with HIV at Cotonou Benin. Mali Med 22: 37-43.

19. Khalili H, Dashti-Khavidaki S, Mohraz M, Etghani A, Almasi F (2009) Antiretroviral induced adverse drug reactions in Iranian human immunodeficiency virus positive patients. Pharmacoepidemiol Drug Saf 18: 848-857.

20. Baudrant M, Bagheri H, Cuzin L, Sommet A, Bassoua V, et al. (2006) Analysis of antiretroviral drugs-induced adverse effects. Therapie 61: 171-176.

21. Duval X, Journot V, Leport C, Chene G, Dupon M, et al. (2004) Incidence of and risk factors for adverse drug reactions in a prospective cohort of $\mathrm{HIV}$-infected adults initiating protease inhibitor-containing therapy.
Clinical infectious diseases: an official publication of the Infectious Diseases Society of America 39: 248-255.

22. Guitton E, Montastruc JL, Lapeyre-Mestre M (2006) French Network of Pharmacovigilance C. Influence of HCV or HBV coinfection on adverse drug reactions to antiretroviral drugs in HIV patients. Eur J Clin Pharmacol 62: 243-249.

23. Modayil RR, Harugeri A, Parthasarathi G, Ramesh M, Prasad R, et al. (2010) Adverse drug reactions to antiretroviral therapy (ART): an experience of spontaneous reporting and intensive monitoring from ART centre in India. Pharmacoepidemiol Drug Saf 19: 247-255.

24. Maiga AI, Sangare SA, Fofana DB, Dao S, Haidara I, et al. (2014) Interest of CD4+T cell count in HIV-infected patients and monitored in the Ségou health district (Mali). Rev Mal Infect Microbiol 2: 22-31.

25. Bennett KK, DeGruttola VG, Marschner IC, Havlir DV, Richman DD (2002) Baseline predictors of CD4 T-lymphocyte recovery with combination antiretroviral therapy. Journal of Acquired Immune Deficiency Syndromes 31: 20-26.

26. World Health Organization (WHO) (2015) Guideline on when to start antiretroviral therapy and on pre-exposure prophylaxis for HIV.

27. Morlat P (2014) Management of HIV-infected persons. Recommendations of the expert group. Paris: Flammarion.

28. CSLS/MS (2013) Policy and protocols for the antiretroviral treatment of HIV/AIDS in Mali. 4. Bamako 2013: 20.

29. Chiffoleau A, Veyrac G, Brunet-François C, Raffi F, Bourin M, et al. (2002) Systematic collection of adverse effects of HIV medicines: 18 months of collaboration between CISIH and CRPV. The Pharmacologist's Letter 16: 123-129.

30. Zannou DM, Denoeud L, Lacombe K, Amoussou-Guenou D, Bashi J, et al. (2009) Incidence of lipodystrophy and metabolic disorders in patients starting non-nucleoside reverse transcriptase inhibitors in Benin. Antiviral therapy 14: 371-380.

31. Cherry CL, Skolasky RL, Lal L, Creighton J, Hauer P, et al. (2006) Antiretroviral use and other risks for HIV-associated neuropathies in an international cohort. Neurology 66: 867-873.

32. Forna F, Liechty CA, Solberg P, Asiimwe F, Were W, et al. (2007) Clinical toxicity of highly active antiretroviral therapy in a home-based AIDS care program in rural Uganda. Journal of acquired immune deficiency syndromes 44: 456-462.

33. Aouizerat BE, Miaskowski CA, Gay C, Portillo CJ, Coggins T, et al. (2010) Risk factors and symptoms associated with pain in HIV-infected adults. J Assoc Nurses AIDS Care 21: 125-133.

34. Zouiten F, Ammari L, Goubantini A, Tiouiri H, Slim A, et al. (2003) Effect of triple antiretroviral therapy on Tunisian AIDS profile: study of 139 cases. Tunis Med 81: 956-962.

35. Pochard L, Hauviller L, Cuzin L, Eyvrard F, Sommet A, et al. (2014) Use of Nadis $((\mathrm{R}))$ software to improve adverse drug reaction reporting of antiretroviral drugs: experience in south west of France (midi-pyrenees). Therapie 69: 149-155.

36. Abdissa SG, Fekade D, Feleke Y, Seboxa T, Diro E (2012) Adverse drug reactions associated with antiretroviral treatment among adult Ethiopian patients in a tertiary hospital. Ethiop Med J 50: 107-113.

37. Coulibaly SM, Oumar AA, Ag Aboubacrine S, Coulibaly S, Koumare BY, et al. (2007) The clinical and biological tolerance of nevirapine among patients with AIDS under treatment at the Hospital of the Point G]. Mali Med 22: 1-4.

38. Namisango E, Harding R, Atuhaire L, Ddungu H, Katabira E, et al. (2012) Pain among ambulatory HIV/AIDS patients: multicenter study of prevalence, intensity, associated factors, and effect. J Pain 13: 704-713.

39. Namme LH, Doualla MS, Choukem SP, Temfack E, Ashuntantang G, et al. (2012) Adverse drug reactions of Highly Active Antiretroviral Therapy (HAART) in HIV infected patients at the General Hospital, Douala, Cameroon: a cross sectional study. Pan Afr Med J 12: 87.

40. Agu KA, Oparah AC (2013) Adverse drug reactions to antiretroviral therapy: Results from spontaneous reporting system in Nigeria. Perspect Clin Res 4: 117-124. 